\title{
Biodiversity of Medicinal Plants Containing Essential Oil and Their Spreading in Adjara
}

\author{
Natela Varshanidze ${ }^{1}$, Nazi Turmanidze ${ }^{1}$, Ketevan Dolidze ${ }^{1}$, Nana Zarnadze ${ }^{1}$, Inga Diasamidze ${ }^{1}$, \\ Tinatin Epitashvili², Tamar Katcharava, \\ ${ }^{1}$ Department of Biology, Faculty of Natural Sciences and Health, Batumi Shota Rustaveli State University, Batumy, Georgia \\ ${ }^{2}$ Biotechnology Center, Georgian Technical University, Tbilisi, Georgia
}

Copyright $\mathrm{C} 2018$ by authors, all rights reserved. Authors agree that this article remains permanently open access under the terms of the Creative Commons Attribution License 4.0 International License

\begin{abstract}
Adjara (South Kolkheti), located in the southwestern part of Georgia, it is characterized by warm and humid climate and subtropical climate, which is due to the proximity of the Black Sea. Adjara is remarkably rich in the diversity of flora not only in Georgia, but throughout the Caucasus. At present there are 1837 plant species in the flora of Adjara, 72 of which contain essential oils, they are distributed in 22 families and in 52 genera. Plants containing essential oil belongs: Lamiaceae - 18 species, Asteraceae-17 types, Apiaceae - 9, Cupressaceae-3, Pinaceae-3, Myrtaceae-3. Vital forms are 7 species of trees, 5 species of bushes, 1 species of tree or bushes, 49 species of perennial grass, 6 species of annual grass. According to the geographical coordinates from the sea level up to 0-500 $\mathrm{m}$ are spread 36 species containing essential oils, from the sea level up to $500-1000 \mathrm{~m} 28$ species, from the sea level up to $1000 \mathrm{~m} 2200 \mathrm{~m} 33$ species. According to the origin of species, 48 species are wild relatives, 7 is cultivated as vegetables, 3 species are introduced, 3 species are invasive. According to the percentage content of essential oils there are: 1 species - 0,025-0,04\%, 4 species - 0,1-0.44\%, 37 species - 0,2-0,4\%, 21 Species - 0,7-1,0\%, 7 species $1,0-3,0 \%, 3$ species $-4,0-6,0 \%$. In the studied species the essential oils structure is complex and diverse. Monotarpens are distributed in 10 species, Sesquitterpaces in 5 species, $\alpha$-pinene in 7 species, $\beta$-pinene in 3 species, $\alpha$ and $\beta$ - pinene in 16 species, cineol in 8 species, menthol in 6 species, lemonene in 3 species, in single species are found geraniol, borneol, apiol, thujone, karvakrol and others. In the studied species has been identified 3 prospective invasive species, which are distinguished by great resource, not used Georgia as per officinal medicine, but in their home countries (USA, Japan, China) are valuable medicinal plants, these species produced oils containing quantitative analysis, analysis revealed that, Artemissia vulgaris of upper part of the ground contain $0,9 \%$ essential oils, with components korizin, tuion, cineol. Perilla nancinensis of upper part of the ground contain $0,88 \%$ essential oils; the main component of the essential oil are perilla-ceton and carophylen, Erygeron canadensis
\end{abstract}

upper part of the ground contain $0,93 \%$ essential oils and its main components are limonene and terpineol. It also contains alcaloyds, flavonoids and tanner materials

Keywords Adjara Flora, Essential Oil, Family, Medicinal Plants, Biodiversity

\section{Introduction}

Adjara is located in the south western part of Georgia. It is characterized by warm and humid subtropical climate. Flora of Adjara is unique, diversity and among them are many endemic. The distinguishing nature of the flora determines that Kolkheti was the shelter-refugee of ancient plant species (relics) during the ice age. Adjara also rich in invincible, invasive and wild relatives species. Adjara is a mountainous region and its flora is characterized by vertical lobes of spread. At present, there are 1837 species registered in the floristic region of Adjara, which include 159 families and 742 genera, from them used for medicinal purposes - 180 species. Among the medicinal plants, there are plants containing essential oils. They contain up to twenty organic compounds, including hydrocarbon mixtures and oxygen-containing components: alcohol, aldehydes, acute esters, phenols, carbonic acids, oxides and lactones. Essential oils are located in special glands, which are located in leaves, flowers, fruits, seeds, roots. The quantitative content of essential oils from $0.01 \%$ to several percent of the plant. Its content depends on the type of plant, on the development stage, on the natural conditions and the plant's age. At the end of vegetation, the content of essential oils are always reduced and collected in the seed. The function of essential oils in the plant is the drawing of insect suppressor, and they regulate the plant's heat transfer. Essential supplements in medicine are used as antibacterial and antimicrobial means, have sedative, spasmolytic and other effects. Thus, the study of plants containing essential 
oils is one of the most important problems.

\section{Method}

The major method of the research is the traditional expedition-excursion method-collecting plants for herbarium and cameral processing. We identified plants according to the plant indexes of Adjara [3.4], Crops international descriptors (International crop descriptors), Peculiarities of crop international collecting (International collecting descriptors) [2.6]. We made the photochemical analysis according to the methods of hydro-distillation and air-liquid chromatography [1].

\section{Results}

Plants spreaded in Adjara and contain essential oils are Pterophyta which is belonged by 1 family with 2 races and 2 species; Pinophyta - 2 families, 4 races and 6 species; Magnoliophyta - 46 races and 64 species of 19 families. The richest families are Lamiaceae - 18 species, Asteraceae - 17 species, Apiaceae - 9 species. 7 species of plant containing essential oil are trees, 1 species - tree or bushes, 5 species of bushes, perennial grasses - 49 and annual grasses - 6 species.

We can find out 4 important species: Artemissia vulgaris, Erygeron Canadensis, Melissa officinalis, Perilla nancinensis.

\section{Discussion}

The above is strengthened by the fact that WWF's initiative on "100 hotpots of European forests" (i.e. 100 unprotected forest plots requiring protection) priority was given to unique Kolkheti relict forests of Adjara [5]. Flora of Adjara includes 1837 wild relative species, which are united in 159 family and 742 genus. Ferns are $61(3,32 \%)$, Gimnospermae - 8 (0,44 \%), Angiospermae 1768 (96,24\%), Dicotiledone are $1372(74,67 \%)$, monocotiledone 396 $(21,55 \%)$; wild relatives of grasses are 1660 species [3.4.7].

According to the analysis of flora of Adjara, we have established the fact that 72 types of plants in Adjara contain essential oils [8.9.10]. 7 species of plant containing essential oil are trees, 1 species - tree or bushes, 5 scpecies of bushes, perennial grasses - 49 and annual grasses - 6 species. With the high diversity of species are distinguished the following families: Lamiaceae - 18, Asteraceae - 17, Apiaceae - 9, Cupressaceae - 3, Pinaceae-3, Myrtaceae-3, Polypodiaceae - 2, Adoxaceae 2, Fabaceae, Acoraceae, Aristolochiaceae, Cyperaceae, Geraniaceae, Oleaceae, Lauraceae, Oleaceae, Liliaceae, Ruscaceae, Primulaceae, Scrophulariaceae, Valerianaceae, Violaceae - 1. There are four species containing important peculiarities: Artemissia vulgaris, Erygeron Canadensis, Melissa officinalis, Perilla nancinensis. Their annotated list and meaning is given in the table 1 .

Table 1. List of plants having essential oils in Adjara

\begin{tabular}{|c|c|c|c|c|c|}
\hline$\#$ & Latin Name & Vital Form & $\begin{array}{c}\text { Distribution } \\
\text { from sea level, } m\end{array}$ & $\begin{array}{c}\text { Part of essential } \\
\text { oils }\end{array}$ & Phytochemical content \\
\hline 1 & $\begin{array}{c}\text { Pterophyta } \\
\text { Family Polypodiaceae } \\
\text { Pteridium tauricum } \\
\end{array}$ & Perennial grass & $50-1700$ & Underground part & $\begin{array}{c}0,025-0,045 \% \text { Essential oils, } \\
\text { carbohydrates, bitter substances, } \\
\text { monotarpens }\end{array}$ \\
\hline 2 & $\begin{array}{l}\text { Family Polypodiaceae } \\
\text { Dryipteris filix mas }\end{array}$ & Perennial grass & $50-1700$ & Underground part & $\begin{array}{c}0,144-0.2 \% \text { Essential oils, tanning } \\
\text { substances, sapons, flavonids, } \\
\text { phenolic compounds, organic acids. } \\
\text { macroelements: } \mathrm{K}, \mathrm{C}, \mathrm{Mg}, \mathrm{Fe} ; \mathrm{Zn}, \mathrm{Se}, \\
\mathrm{Ba}, \mathrm{Al}, \mathrm{Fe}\end{array}$ \\
\hline 3 & $\begin{array}{c}\text { Pinophyta } \\
\text { Family Cupressaceae } \\
\text { Pinophyta } \\
\text { Juniperus Sabina }\end{array}$ & Evergreen bush & $1700-2200$ & $\begin{array}{l}\text { Needle, } \\
\text { buds }\end{array}$ & $\begin{array}{c}0,16-0,44 \% \text { Essential oils, sugar, } \\
\text { phinephoside, fatty oils, apple acids, } \\
\text { antibiotic, biciccular }\end{array}$ \\
\hline 4 & $\begin{array}{c}\text { Family Cupressaceae } \\
\text { Juniperus rufescens } \\
\text { Link } \\
\end{array}$ & Evergreen bush & $1700-2200$ & Cones & $\begin{array}{c}0,21-0,43 \% \text { Essential oils, vitamine } C, \\
\text { phytocytes, fatty oils, organic acids, } \alpha \\
\text {-pinen, cedrol }\end{array}$ \\
\hline 5 & $\begin{array}{l}\text { Family Cupressaceae } \\
\text { Juniperus communis }\end{array}$ & Evergreen bush & $1200-1450$ & Cones & $\begin{array}{c}0,21-0,42 \% \text { Essential oils, fatty oils, } \\
\text { vitamin C, organic acids, } \alpha \text {-pinen } \\
27.2 \%, \beta \text {-pinen } 22,4 \%, 3 \text {-Karen } 7 \%, \\
\beta \text {-felandren } 5,7 \% .\end{array}$ \\
\hline 6 & $\begin{array}{c}\text { Family Pinaceae } \\
\text { Abies nordmaniana }\end{array}$ & Evergreen tree & $1800-2050$ & Cones & $\begin{array}{c}0,19-0,4 \% \text { Essential oils, resins, } \\
\text { phytochemicals, vitamins C and B, } \\
\alpha \text {-tuion, } \\
\beta \text { - tuion, } 1,4 \text {-cineol, cisocimen, } \\
\alpha \text {-pinen, } \beta \text {-pinen, terpinen, metiltimol, } \\
\text { longiciklen, izoborneol }\end{array}$ \\
\hline 7 & $\begin{array}{l}\text { Family Pinaceae } \\
\text { Picea orientalis }\end{array}$ & Evergreen tree & $800-2100$ & $\begin{array}{l}\text { Needle, } \\
\text { buds, } \\
\text { cones }\end{array}$ & $\begin{array}{l}0,2-0,4 \% \text { Essential oils, phinephoside, } \\
\text { resins, salts, } \alpha \text {-pinen, karen, kampen, } \\
\text { mirceen, fellandren, myrcen, } \\
\text { terminien, cineol, } \mathrm{Cr}, \mathrm{Mn}, \mathrm{Cu}, \mathrm{Al},\end{array}$ \\
\hline
\end{tabular}




\begin{tabular}{|c|c|c|c|c|c|}
\hline 8 & $\begin{array}{l}\text { Family Pinaceae } \\
\text { Pinus sosnovskii }\end{array}$ & Evergreen tree & $500-1100$ & Needle, buds & $\begin{array}{l}0,36 \% \text { Essential oils tanning agents, } \\
\text { vitamin } C \text {, carotenoids, flavonoids, } \alpha \\
\text {-pinen, } \beta \text { - pinen } 40 \%, \beta \text {-lemonen } 40 \%\end{array}$ \\
\hline 9 & $\begin{array}{l}\text { Magnoliophyta } \\
\text { Family Adoxaceae } \\
\text { Sambucus ebulus }\end{array}$ & Perennial grass & $50-2200$ & Fruits & $\begin{array}{c}0.32 \% \text {, essential oils, tissue } \\
\text { substances, bitter substances, organic } \\
\text { acids }\end{array}$ \\
\hline 10 & $\begin{array}{c}\text { Family Apiaceae } \\
\text { Anethum graveolens }\end{array}$ & Perennial grass & $50-2000$ & $\begin{array}{l}\text { Upper part of } \\
\text { ground }\end{array}$ & $\begin{array}{l}0,2-0.4 \% \text { Sensivitopenic essential oils, } \\
\text { Vitamins C and B. }\end{array}$ \\
\hline 11 & $\begin{array}{c}\text { Family Apiaceae } \\
\text { Anethum graveolens }\end{array}$ & Perennial grass & $50-2000$ & $\begin{array}{l}\text { Upper part of } \\
\text { ground }\end{array}$ & 0,2-0.4\% Essential oils, vitamins C, B, \\
\hline 12 & $\begin{array}{l}\text { Family Apiaceae } \\
\text { Angelica adjarica }\end{array}$ & Perennial grass & $1200-1700$ & $\begin{array}{l}\text { Upper part of } \\
\text { ground }\end{array}$ & $\begin{array}{c}0.1-0.2 \% \text { Essential oils, bitter } \\
\text { substances, alkaloids }\end{array}$ \\
\hline 13 & $\begin{array}{l}\text { Family Apiaceae } \\
\text { Apium graveolens }\end{array}$ & Long-standing grass & $50-2000$ & $\begin{array}{l}\text { All parts of the } \\
\text { plant }\end{array}$ & $\begin{array}{l}0,1 \% \text { essential oils, } 3 \% \text { sugars, } \\
\text { glycosides, mucous, potassium, } \\
\text { calcium, phosphorus and sodium salts, } \\
\text { acidic acid, vitamins C, B, PP, } \\
\text { glycosides, limonen (up to } 70 \% \text { ), } \\
\text { terpenic hydrocarbons }\end{array}$ \\
\hline 14 & $\begin{array}{l}\text { Family Apiaceae } \\
\text { Carum carvi }\end{array}$ & Perennial grass & $50-1000$ & $\begin{array}{l}\text { Upper part of } \\
\text { ground }\end{array}$ & $\begin{array}{c}1 \% \text { essential oils, fats, protein } \\
\text { substances, carvone } 45-60 \% \text {, } \\
\text { carvacrol, limonen }\end{array}$ \\
\hline 15 & $\begin{array}{l}\text { Family Apiaceae } \\
\text { Coriadrum sativum }\end{array}$ & Annual grass & $50-2000$ & $\begin{array}{l}\text { Upper part of } \\
\text { ground }\end{array}$ & $\begin{array}{c}0,7-1 \% \text { Essential oils, fats, } 92 \% \\
\text { glycerides, } 7.5 \% \text { unconventional } \\
\text { substances, alkaloids, vitamins A and } \\
\text { C. }\end{array}$ \\
\hline 16 & $\begin{array}{l}\text { Family Apiaceae } \\
\text { Foeniculum vulgare }\end{array}$ & Annual grass & $50-2000$ & $\begin{array}{l}\text { Upper part of } \\
\text { ground }\end{array}$ & $\begin{array}{l}\text { The fruits contain } 4-6.5 \% \text { essential } \\
\text { oils, } 12-18 \% \text { fats. The leaves contain } \\
0,62-1,54 \% \text { essential oils, vitamins } \mathrm{C} \text {, } \\
\text { B, nicotine and amber acid, mineral } \\
\text { salts, microelements: } \mathrm{Ca}, \mathrm{K}, \mathrm{P} \text {, seeds } \\
\text { contain up to } 18 \% \text { fatty oil. }\end{array}$ \\
\hline 17 & $\begin{array}{l}\text { Family Apiaceae } \\
\text { Petroselinum sativum }\end{array}$ & Annual grass & $50-2000$ & All parts & $\begin{array}{l}\text { The roots contain } 0,1 \% \text { of essential } \\
\text { oils, mucus, microelements: } \\
\text { potassium, calcium, iron, phosphorus. } \\
\text { Seeds contain essential oils, fatty oils, } \\
\text { vitamin C and pro-vitamin A. Leaves } \\
\text { contain, tannins, ascorbic acid, } \\
\text { difficult essential oils, flavonoids, } \\
\text { mineral salts, microelements, vitamin } \\
\text { K }\end{array}$ \\
\hline 18 & $\begin{array}{l}\text { Family Apiaceae } \\
\text { Peucedanum } \\
\text { caucasicum (Bieb.) }\end{array}$ & Perennial grass & $1200-1500$ & $\begin{array}{l}\text { Upper part of } \\
\text { ground }\end{array}$ & $\begin{array}{l}\text { 0,7-1\% Essential oils, protein } \\
\text { substances }\end{array}$ \\
\hline 19 & $\begin{array}{c}\text { Family Apiaceae } \\
\text { Trachyspermum ammi }\end{array}$ & Perennial grass & $400-1000$ & $\begin{array}{l}\text { Upper part of } \\
\text { ground }\end{array}$ & $\begin{array}{l}0,2-0.4 \% \text { Essential oils, glycosides, } \\
\text { flavonoids, } 20 \% \text { fats, } \alpha \text { - pinen }\end{array}$ \\
\hline 20 & $\begin{array}{l}\text { Family Acoraceae } \\
\text { Acorus calamus }\end{array}$ & Perennial grass & $0-25$ & Radicel & $\begin{array}{c}1-2,2 \% \text { of essential oils, bitter } \\
\text { glycoside, ascorbic acid ( } 150 \mathrm{mg} / \%), \\
\text { tonsillitis, alkaloids }\end{array}$ \\
\hline 21 & $\begin{array}{c}\text { Family } \\
\text { Aristolochiaceae } \\
\text { Asarum intermedium } \\
\text { (C.A.Mey.) Grossh } \\
\end{array}$ & Perennial grass & $500-700$ & $\begin{array}{l}\text { Upper part of } \\
\text { ground }\end{array}$ & $\begin{array}{l}0,2-0.4 \% \text { Essential oils, alkaloids, } \\
\text { phenols, } \beta \text {-pinen, } \beta \text {-filandren }\end{array}$ \\
\hline 22 & $\begin{array}{l}\text { Family Asteraceae } \\
\text { Achillea bisserata }\end{array}$ & Perennial grass & $400-1200$ & Flowers & $\begin{array}{c}0,85 \% \text { essential oils, alkaloid, } \\
\text { amylene, inuline, asparagine, nitrates, } \\
\text { organic acids, carotene, vitamins } \mathrm{C}, \mathrm{K} \text {, } \\
\text { B }\end{array}$ \\
\hline 23 & $\begin{array}{l}\text { Family Asteraceae } \\
\text { Achillea bieberteinii }\end{array}$ & Perennial grass & $500-2000$ & Flowers & $\begin{array}{c}0.83 \% \text { Essential oils, alkaloid } \\
\text { amylene, inuline, asparagine, nitrates, } \\
\text { organic acids, carotene, vitamins C, K, } \\
\text { B }\end{array}$ \\
\hline 24 & $\begin{array}{l}\text { Family Asteraceae } \\
\text { Achillea nobilis }\end{array}$ & Perennial grass & $500-2000$ & Flowers & $\begin{array}{c}0,84 \% \text { essential oils, inulin, nitrates, } \\
\text { organic acids, carotene, vitamins } \mathrm{C}, \mathrm{K} \text {, } \\
\text { B,A, sabinen }\end{array}$ \\
\hline 25 & $\begin{array}{l}\text { Family Asteraceae } \\
\text { Achillea neilreichi }\end{array}$ & Perennial grass & $500-2000$ & Flowers & $\begin{array}{c}0,7 \% \text {, essential oils, alkaloide } \\
\text { amylene, inulin, asparagine, nitrates, } \\
\text { organic acids, carotene, vitamins C, K, } \\
\alpha \text {-pinen, sabinen }\end{array}$ \\
\hline
\end{tabular}




\begin{tabular}{|c|c|c|c|c|c|}
\hline 26 & $\begin{array}{c}\text { Family Asteraceae } \\
\text { Achillea fillipendullina }\end{array}$ & Perennial grass & $500-2000$ & Flowers & $\begin{array}{c}0,6 \% \text { essential oils, alkaloids, } \\
\text { flavonoid, rutin, inulin, asparagine, } \\
\text { nitrates, organic acids, vitamins } \mathrm{C}, \mathrm{K} \text {, } \\
\mathrm{B}\end{array}$ \\
\hline 27 & $\begin{array}{c}\text { Family Asteraceae } \\
\text { Achillea latiloba }\end{array}$ & Perennial grass & $500-2000$ & Flowers & $\begin{array}{c}0,72 \% \text { essential oils, alkaloid amylene, } \\
\text { flavonoids, apple acid, carotene, } \\
\text { vitamins } \mathrm{C}, \mathrm{K} \text {, sabinen }\end{array}$ \\
\hline 28 & $\begin{array}{c}\text { Fam. Asteraceae } \\
\text { Achillea millefolium }\end{array}$ & Perennial grass & $500-2000$ & Flowers & $\begin{array}{c}0,85 \% \text { essential oils, alkaloid, } \\
\text { amylene, flavonoids, apple acid, } \\
\text { carotene, vitamins C, K, } \alpha \text {-pinen } \\
(3.6-8.0), \beta \text {-pinen }(18.4-33.9)\end{array}$ \\
\hline 29 & $\begin{array}{l}\text { Family Asteraceae } \\
\text { Achillea satacea }\end{array}$ & Perennial grass & $50-800$ & Flowers & $\begin{array}{c}\text { 0,9\% essential oils, alkaloid, amylene, } \\
\text { flavonoids, apple acid, carotene, } \\
\text { vitamin } \mathrm{C}, \alpha \text {-pinene, sabinene, } \\
\beta \text {-pinene }\end{array}$ \\
\hline 30 & $\begin{array}{l}\text { Family Asteraceae } \\
\text { Artemisia absinthum }\end{array}$ & Perennial grass & $50-200$ & $\begin{array}{l}\text { upper part of } \\
\text { ground }\end{array}$ & $\begin{array}{c}0,72 \% \text { essential oils, alkaloid, } \\
\text { amylene, flavonoids, apple acid, } \\
\text { carotene, vitamins C, K }\end{array}$ \\
\hline 31 & $\begin{array}{l}\text { Family Asteraceae } \\
\text { Artemisia vulgaris }\end{array}$ & Perennial grass & $50-1800$ & $\begin{array}{l}\text { upper part of } \\
\text { ground }\end{array}$ & $\begin{array}{c}0,8 \% \text { Essential oils, tanning and } \\
\text { mucous substances, sugars, carotene, } \\
\text { ascorbic acid }\end{array}$ \\
\hline 32 & $\begin{array}{l}\text { Fam. Asteraceae } \\
\text { Erigeron Canadensis }\end{array}$ & Perennial grass & $50-2000$ & $\begin{array}{l}\text { upper part of } \\
\text { ground }\end{array}$ & $\begin{array}{c}1,1 \% \text { Essential oils, alkaloids, } \\
\text { flavonids, monotarpens, tonsils, } \\
\text { sesquitterpaces, sabinen }\end{array}$ \\
\hline 33 & $\begin{array}{c}\text { Family Asteraceae } \\
\text { Helicrysum graveolens }\end{array}$ & Perennial grass & $2000-2200$ & Flowers & $\begin{array}{l}\text { 0,2-0.4\% Essential oils, flavones and } \\
\text { flavono glycosides, saponins, sterin, } \\
\text { carotene, vitamins } \mathrm{C} \text { and } \mathrm{K} \text {, sodium, } \\
\text { potassium, iron salts, monotarpens: } \\
\alpha \text {-pinene, } \beta \text {-pinene, limonene, } \\
\text { sesquiterpens }\end{array}$ \\
\hline 34 & $\begin{array}{l}\text { Family Asteraceae } \\
\text { Helicrysum } \\
\text { poliphylum }\end{array}$ & Perennial grass & $2000-2200$ & Flowers & $\begin{array}{c}\text { 0,2-0,4\% Ethers, flavones and } \\
\text { flavonogenic glycosides, saponins, } \\
\text { sterin, carotene, vitamins C and } \mathrm{K} \text {, } \\
\text { tanning substances, microelements, } \\
\text { cadinene, monotarpens }\end{array}$ \\
\hline 35 & $\begin{array}{l}\text { Family Asteraceae } \\
\text { Inula helenium }\end{array}$ & Perennial grass & $1700-2100$ & Underground part & $\begin{array}{l}\text { 0,2-0,4\% essential oils, lactones, } \\
\text { inulin, starch, polysaccharides, } \\
\text { pectinous substances, resinous } \\
\text { substances, vitamin E, ash, pinene, } \\
\text { sabinene }\end{array}$ \\
\hline 36 & $\begin{array}{c}\text { Family Asteraceae } \\
\text { Matricaria chamomilla } \\
\text { var. recuitita }\end{array}$ & Annual grass & $600-1000$ & Flowers & $\begin{array}{l}0,8 \% \text { - Essential oils, lactones, } \\
\text { matricarin, organic acids, resins, } \\
\text { polysaccharides, flavonoids, } \\
\text { carotenoids, ascorbic acid }\end{array}$ \\
\hline 37 & $\begin{array}{c}\text { Family Asteraceae } \\
\text { Pyretrum } \\
\text { parthenifolium }\end{array}$ & Annual grass & $1500-1800$ & Flowers & $\begin{array}{c}0,6 \% \text { Essential oils, lactones, } \\
\text { matricarin, organic acids, resins, } \\
\text { polysaccharides, flavonoids, } \\
\text { carotenoids, ascorbic acid }\end{array}$ \\
\hline 38 & $\begin{array}{l}\text { Family Asteraceae } \\
\text { Pyretrum roseum }\end{array}$ & Perennial grass & $600-1000$ & Flowers & $\begin{array}{c}0,8 \% \text { Essential oils, matricarin, } \\
\text { organic acids, resins, flavonoids, } \\
\text { polysaccharides, carotenoids, ascorbic } \\
\text { acid }\end{array}$ \\
\hline 39 & $\begin{array}{c}\text { Family Brassicaceae } \\
\text { Capsella bursa-pastoris }\end{array}$ & Annual grass & $50-2000$ & $\begin{array}{l}\text { upper part of } \\
\text { ground }\end{array}$ & $\begin{array}{l}\text { 0.16-0,44\% Essentials, glycosides, } \\
\text { saponins, alkaloid. wine, apple and } \\
\text { lemon acids, vitamin C ( } 200 \mathrm{mg} / \%) \text {, } \\
\text { carotene, mineral salts, iciccular }\end{array}$ \\
\hline 40 & $\begin{array}{l}\text { Family Brassicaceae } \\
\text { Raphanus sativus }\end{array}$ & Annual grass & $25-500$ & Underground part & $\begin{array}{c}\text { 0,2-0.4\% Essential oils, A, B, C } \\
\text { vitamins, organic acids, simple } \\
\text { carbohydrates, sesquiterpens }\end{array}$ \\
\hline 41 & $\begin{array}{l}\text { Family Cyperaceae } \\
\text { Cyperus badius }\end{array}$ & Perennial grass & $23-500$ & Underground part & $\begin{array}{c}0,23-0,45 \% \text { Essential substances, } \\
\text { coumarines, } \beta \text { - selinen, } \alpha \text {-cyperon, } \\
\text { cyperen, }\end{array}$ \\
\hline 42 & $\begin{array}{l}\text { Family Fabaceae } \\
\text { Trifolium pretense }\end{array}$ & Perennial grass & $25-1900$ & $\begin{array}{l}\text { Upper part of } \\
\text { ground }\end{array}$ & $\begin{array}{l}\text { 0,24-0,44\% Essential oils, phenols, } \\
\text { carbohydrates, monotarpens, } \\
\text { isoprenoid }\end{array}$ \\
\hline 43 & $\begin{array}{l}\text { Family Geranidaceae } \\
\text { Geranium robertianum }\end{array}$ & Perennial grass & $1000-2200$ & $\begin{array}{l}\text { Upper part of } \\
\text { ground }\end{array}$ & $\begin{array}{c}0,2-0.4 \% \text { Essential oils, alkaloids, } \\
\text { phenols, isoprenoid }\end{array}$ \\
\hline
\end{tabular}




\begin{tabular}{|c|c|c|c|c|c|}
\hline 44 & $\begin{array}{c}\text { Fam. Lamiaceae } \\
\text { Calamintha grandiflora }\end{array}$ & Perennial grass & $1000-2200$ & $\begin{array}{l}\text { Upper part of } \\
\text { ground }\end{array}$ & $\begin{array}{c}0,3-0.8 \% \text { Essential oils, carbohydrates, } \\
\text { flavonoids }\end{array}$ \\
\hline 45 & $\begin{array}{c}\text { Family Lamiaceae } \\
\text { Glechoma hederaceae }\end{array}$ & Perennial grass & $50-2000$ & $\begin{array}{l}\text { Upper part of } \\
\text { ground }\end{array}$ & $\begin{array}{c}0,2-0,4 \% \text { Essential oils, } 8 \% \text { tannins, } \\
\text { bitter substances, choline }\end{array}$ \\
\hline 46 & $\begin{array}{l}\text { Family Lamiaceae } \\
\text { Lavandula vera }\end{array}$ & Evergreen bush & $30-100$ & $\begin{array}{l}\text { Upper part of } \\
\text { ground }\end{array}$ & $\begin{array}{l}0162-0.38 \% \text { Essential oils, organic } \\
\text { acids, carotine, flavonoid hepperidine }\end{array}$ \\
\hline 47 & $\begin{array}{l}\text { Family Lamiaceae } \\
\text { Leonurus } \\
\text { quinquelobatus Gilib }\end{array}$ & Perennial grass & $1600-1900$ & $\begin{array}{l}\text { Upper part of } \\
\text { ground }\end{array}$ & $\begin{array}{c}0,2-0.4 \% \text { Essential oils, alkaloids, } \\
\text { tanning agents, flavonoids, } \alpha \text {-pinen, } \\
\text { piperiton }\end{array}$ \\
\hline 48 & $\begin{array}{l}\text { Family Lamiaceae } \\
\text { Mellisa officinalis }\end{array}$ & Perennial grass & $25-1000$ & $\begin{array}{l}\text { Upper part of } \\
\text { ground }\end{array}$ & $\begin{array}{c}0,2 \% \text { Essential oils, tissue substances, } \\
\text { mucus, cytral }\end{array}$ \\
\hline 49 & $\begin{array}{l}\text { Family Lamiaceae } \\
\text { Mentha aquatica }\end{array}$ & Perennial grass & $500-2400$ & $\begin{array}{l}\text { Upper part of } \\
\text { ground }\end{array}$ & $\begin{array}{l}0,4-0.8 \% \text { Essential oils, carbohydrates, } \\
\text { glycosides, } \alpha \text {-pinen, } \beta \text { - pinen, piperiton, } \\
\text { menthol, terpinen, menthofuran }\end{array}$ \\
\hline 50 & $\begin{array}{l}\text { Family Lamiaceae } \\
\text { Mentha longifolia }\end{array}$ & Perennial grass & $500-2400$ & $\begin{array}{l}\text { Upper part of } \\
\text { ground }\end{array}$ & $\begin{array}{l}0,3-0.8 \% \text { Essential oils, flavonids, } \alpha- \\
\text { pinen, } \beta \text {-pinen, terpinen }\end{array}$ \\
\hline 51 & $\begin{array}{l}\text { Family Lamiaceae } \\
\text { Mentha pulegium }\end{array}$ & Perennial grass & $50-1000$ & $\begin{array}{l}\text { Upper part of } \\
\text { ground }\end{array}$ & $\begin{array}{c}\text { 0,2-0.4\% Essential oils, vitamins C, B, } \\
\text { chromotids, simple carbohydrates, } \\
\text { menthol, limonene }\end{array}$ \\
\hline 52 & $\begin{array}{l}\text { Family Lamiaceae } \\
\text { Mentha piperita }\end{array}$ & Perennial grass & $50-200$ & $\begin{array}{l}\text { Upper part of } \\
\text { ground }\end{array}$ & $\begin{array}{l}2,5 \%-4,6 \% \text { Essential oils, } 40-70 \% \\
\text { organic acids, carotine and flavonides, } \\
\text { hepperidine, } \alpha \text {-pinen, } \beta \text {-pinen, } \\
\text { terpinene, menthol } 40-70 \%\end{array}$ \\
\hline 53 & $\begin{array}{l}\text { Family Lamiaceae } \\
\text { Origanum vulgare }\end{array}$ & Perennial grass & $200-500$ & $\begin{array}{l}\text { Upper part of } \\
\text { ground }\end{array}$ & $\begin{array}{c}0,5-1,2 \% \text { Essential oils, tissue } \\
\text { substances, vitamin C } 565 \mathrm{mg} / \% \\
\text { carvacrol, menthol }\end{array}$ \\
\hline 54 & $\begin{array}{l}\text { Family Lamiaceae } \\
\text { Osimum basilicum }\end{array}$ & Annual grass & $50-1000$ & $\begin{array}{l}\text { Upper part of } \\
\text { ground }\end{array}$ & $\begin{array}{c}0,2-0,4 \% \text { of essential oils, tannins, } \\
\text { difficult carbohydrates, carotene, } \\
\text { vitamins C, B2, K, linalool (17.7 \%), } \\
\text { methyl chavicol }(28.0 \%) \text { and eugenol } \\
\text { (36.2 \%) }\end{array}$ \\
\hline 55 & $\begin{array}{l}\text { Family Lamiaceae } \\
\text { Perillana nankinensis }\end{array}$ & Perennial grass & $30-600$ & $\begin{array}{l}\text { Upper part of } \\
\text { ground }\end{array}$ & $\begin{array}{l}0,21-0,42 \% \text { essential oils, flavonoids, } \\
\text { procourmaries, carbohydrates, }\end{array}$ \\
\hline 56 & $\begin{array}{l}\text { Family Lamiaceae } \\
\text { Salvia glutinosa }\end{array}$ & Perennial grass & $400-1200$ & $\begin{array}{l}\text { Upper part of } \\
\text { ground }\end{array}$ & $\begin{array}{l}0,23-0,44 \% \text { Essential oils, organic } \\
\text { acids, carotenoids, } \alpha \text {-pinen, Sabinen, } \\
\beta \text {-pinen, limonene, terpinene }\end{array}$ \\
\hline 57 & $\begin{array}{l}\text { Family Lamiaceae } \\
\text { Salvia solarea }\end{array}$ & Perennial grass & $1100-15000$ & $\begin{array}{l}\text { Upper part of } \\
\text { ground }\end{array}$ & $\begin{array}{l}1,2 \% \text { essential oils, alkaloids, tissue } \\
\text { substances, organic acids, vitamin B, } \\
\text { pinene, linalool, thymol, carvacrol, }\end{array}$ \\
\hline 58 & $\begin{array}{l}\text { Family Lamiaceae } \\
\text { Satureia laxifloraL. }\end{array}$ & Perennial grass & $200-1300$ & $\begin{array}{l}\text { Upper part of } \\
\text { ground }\end{array}$ & $\begin{array}{l}0,17-0.41 \% \text { Essential oils, } \\
\text { procourmaries, ascorbic acid, terpinen, } \\
\text { thymol, carvacrol, microelements, }\end{array}$ \\
\hline 59 & $\begin{array}{l}\text { Family Lamiaceae } \\
\text { Scutellaria galericulata }\end{array}$ & Perennial grass & $500-1700$ & $\begin{array}{l}\text { Upper part of } \\
\text { ground }\end{array}$ & $\begin{array}{c}0,2-0.4 \% \text { Essential oils, } \\
\text { procourmaries, ascorbic acid, } \\
\text { carbohydrates, carvacrol, } \\
\text { microelements, }\end{array}$ \\
\hline 60 & $\begin{array}{l}\text { Family Lamiaceae } \\
\text { Thymus grossheimi }\end{array}$ & Perennial grass & $500-1700$ & $\begin{array}{c}\begin{array}{c}\text { Upper part of } \\
\text { ground }\end{array} \\
\end{array}$ & $\begin{array}{c}0,17-0,37 \% \text { Essential oils, flavonids, } \\
\text { 1,8-cineole }\end{array}$ \\
\hline 61 & $\begin{array}{l}\text { Family Lamiaceae } \\
\text { Stachys sylvatica }\end{array}$ & Perennial grass & $600-1700$ & $\begin{array}{c}\text { Upper part of } \\
\text { ground }\end{array}$ & $\begin{array}{l}0,2-0.4 \% \text { Essential oils, flavonids, } \\
\text { procourmaries, mineral salts. }\end{array}$ \\
\hline 62 & $\begin{array}{l}\text { Family Lauraceae } \\
\text { Laurus nobilis }\end{array}$ & Evergreen tree or bush & $50-1000$ & Leaf & $\begin{array}{l}4-5 \% \text { Essential oils, organic acids, } \\
\text { polysaccharides, } 1,8 \text {-cineol }(40 \%) \text {, } \\
\text { pinen, linalool, limonene, eugenol, }\end{array}$ \\
\hline 63 & $\begin{array}{l}\text { Family Liliaceae } \\
\text { Allium ursinum }\end{array}$ & Perennial grass & $130-1900$ & Bulbs, leaves & $\begin{array}{l}0,2-0.4 \% \text { Essential Oils, vitamins C } \\
\text { and B, phinocytes }\end{array}$ \\
\hline 64 & $\begin{array}{l}\text { Family Ruscaceae } \\
\text { Ruscus ponticus }\end{array}$ & Perennial grass & $400-1000$ & $\begin{array}{c}\text { Upper part of } \\
\text { ground }\end{array}$ & $\begin{array}{c}0,2-0.4 \% \text { Essential oils, sucrose, } \\
\text { monotarpens. }\end{array}$ \\
\hline 65 & $\begin{array}{l}\text { Family Myrtaceae } \\
\text { Eucaliptus cinerea }\end{array}$ & Evergreen tree & $50-300$ & leaves & $\begin{array}{l}2,5 \% \text { essential oils, tannins, } \\
\text { procourmaries, organic acids, cineol, } \\
\text { aldehydes }\end{array}$ \\
\hline 66 & $\begin{array}{l}\text { Family Myrtaceae } \\
\text { Eucaliptus globules }\end{array}$ & Evergreen tree & $50-300$ & leaves & $\begin{array}{l}2,7 \% \text { Essential oils, tannins, } \\
\text { procourmaries, organic acids, cineol, } \\
\text { aldehydes, }\end{array}$ \\
\hline 67 & $\begin{array}{l}\text { Family Myrtaceae } \\
\text { Eucalyptus wiminalis }\end{array}$ & Evergreen tree & $50-300$ & leaves & $\begin{array}{c}2,5 \% \text { essential oils, tannins, } \\
\text { procourmaries, organic acids, cineol, } \\
\text { aldehydes, }\end{array}$ \\
\hline
\end{tabular}




\begin{tabular}{|c|c|c|c|c|c|}
\hline 68 & $\begin{array}{l}\text { Family Oleaceae } \\
\text { Jasminum officinale }\end{array}$ & Crawling bush & $50-500$ & Flower & $\begin{array}{c}\text { 0,2-0.4\% Essential oils, carotenoids, } \\
\text { benzylacetate, benzyl alcohol, } \\
\text { indoline, krezol, vitamin A, B, } \\
\text { 4-terpineol }\end{array}$ \\
\hline 69 & $\begin{array}{c}\text { Family Primulaceae } \\
\text { Primula sibthropii }\end{array}$ & Perennial grass & $50-1000$ & $\begin{array}{l}\text { Upper part of } \\
\text { ground }\end{array}$ & $\begin{array}{l}\text { 0,2-0.4\% Essential oils, carotenoids, } \\
\text { sapons, vitamins C, B, cineol, } \\
\text { aldehydes, mineral salts, }\end{array}$ \\
\hline 70 & $\begin{array}{c}\text { Family } \\
\text { Scrophulariaceae. } \\
\text { Linaria vulgaris }\end{array}$ & Perennial grass & $1700-2300$ & $\begin{array}{l}\text { Upper part of } \\
\text { ground }\end{array}$ & $\begin{array}{l}0,2-0.4 \% \text { Essential oils, flavonoids, } \\
\text { alkaloids. } \alpha \text {-pinen, limonen, piperiton, } \\
\text { microelements }\end{array}$ \\
\hline 71 & $\begin{array}{l}\text { Family Valerianaceae } \\
\text { Valeriana eriophylla }\end{array}$ & Perennial grass & $2000-2100$ & Underground part & $\begin{array}{c}0,19-0.36 \% \text { Essential oils, alkaloids, } \\
\text { tannins, sugars, } \alpha \text {-pinen, limonen, } \\
\text { piperiton, cymol }\end{array}$ \\
\hline 72 & $\begin{array}{l}\text { Family Violaceae } \\
\text { Viola arvensis Murr }\end{array}$ & Perennial grass & $50-2000$ & $\begin{array}{l}\text { Upper part of } \\
\text { ground }\end{array}$ & $\begin{array}{c}\text { Essential oils, flavonoid, carotenoids, } \\
\text { polysaccharides, mucous substances, } \\
\text { isobutanol, } \\
\text { butanol }\end{array}$ \\
\hline
\end{tabular}

From the table 1, there is evident that 50 species contain essential oils are medical; they are used as sedative and digestive system of means. 17 - species have decorative quality, 13 - species are used as food, 9 - species are weeds, the essential oils are piling up in the ground parts and in the 38 species - in upper parts of the ground, 12 - in the flowers, 8 - in the leaves and 4 - species in the fruits.

\section{Conclusions}

In above mentioned species spread in the flora of Adjara we can distinguish 4 species having important peculiarities: Artemissia vulgaris, Erygeron Canadensis, Melissa officinalis, Perilla nancinensis. In order to study the content of essential oils of these species, we made the qualitative and quantitative photochemical analysis; afterwards we proved that: Melissa officinalis (upper ground part) contain 0,02-0,03\% essential oils; Artemissia vulgaris (upper part of the ground) contain $0,5-0,7 \%$ essential oils; Perilla nancinensis (upper part of the ground) contain 0,28\% essential oils; Erygeron canadensis (upper part of the ground) contain $0,33-0,66 \%$ essential oils.

\section{REFERENCES}

[1] Gogia V. (1979). Biochemistry of Subtropical plants. Tbilisi.160p.
[2] Delfan B. Bahmani M, Hassanzadazar H, Saki K, Rafieian-Kopaei M.Rashidipour, M. Bagheri, F, Sharifi, A. Ethnobotany. (1990). Study of effective medicinal plants on gastric problems in Lorestan province, West of Iran. Journal of Chemical and Pharmaceutical Research. (2). 2015. pp. 483-492

[3] Dmitrieva A. (1990). Identify the plant of Adjara, V.I,II.

[4] Identify the plant of Georgia. (1964, 1969). V.I,II. Tbilisi.

[5] Manvelidze Z. Memiadze N.Kharazishvili D. Varshanidze N., Diversityof flora lareaof Adjara. (Listof wild grown plants species). Annalis of Agrarian science, vol.8, No2, Tbilisi. 2010. pp. 93-164.

[6] Puri K. Ethnobotany in the New Europe: People, Health and Wild Plant Resources. Oxford, 2013. 92p.

[7] Varshanidze N. A Specific diversity of medical plants spread in Adjara. Publishing house "Batumi University", Batumi, 2013. 192p.

[8] Kacharava T. (2015) - Sustainable Use Genetic Resources if Medicinal, Aromatic, Spicy, Poisonous Plants, International Conference, "Applied Ecology: Problems, Innovations" Tbilisi, ISBN 978-9941-0-7644-2, http://icae-2015.tsu.ge,p p.241-246;

[9] Kacharava T. (2009) - Medicine, Aromatic, Spicery and Poisonous Plants - Text-book, Publishing house, "universal", ISBN 978-9941-12-575-1, Tbilisi, 188 p.

[10] Kacharava T., Epitashvili T. (2016) - Medicinal, aromatic and spice plants' genetic resources, protection in Georgia, Sustainable, Utilization of Plant Genetic Resources for Agriculture and Food, International scientific conference, Pie-št'any, Slovak Republic, 2016, pp. 34. http://www.vurv.sk/conference/. 